\title{
SEROPREVALENCE OF HUMAN CYSTICERCOSIS IN MAPUTO, MOZAMBIQUE
}

\author{
MANUELA VILHENA, MANUELA SANTOS, AND JORGE TORGAL \\ Centro de Investigação em Saúde Comunitária, Fundação para a Ciência e a Technologia, Lisbon, Portugal; Disciplina de \\ Neurologia Faculdade de Medicina, Universidade Eduardo Mondlane, Maputo, Mozambique; Departamento de Saúde Pública, \\ Faculdade de Ciências Médicas, Universidade Nova de Lisbon, Lisbon, Portugal
}

\begin{abstract}
We carried out a serosurvey for cysticercosis among people visiting the Central Hospital of Maputo, the capital of Mozambique, between January and June 1993. A standardized questionnaire was designed to obtain information on demographic, socioeconomic, environmental, and behavioral characteristics related to the transmission of the infection. Four hundred eighty-nine individuals were tested for anti-cysticercosis antibodies: 222 blood donors and patients from the Department of Orthopedics, 148 patients from the Department of Neurology, and 119 patients from the Department of Psychiatry. The overall positivity rate was $12.1 \%$ (59 of 489). Anti-cysticercus antibodies was detected in $14.9 \%$ of the blood donors and patients from the Department of Orthopedics, $11.5 \%$ of the patients from the Department of Neurology, and $7.6 \%$ of the patients from the Department of Psychiatry. Living in poor sanitary conditions seems to be an important factor related to human cysticercosis in Maputo, Mozambique.
\end{abstract}

Cysticercosis shows clinical symptoms ranging from mild headaches to mental disorders, seizures, changes in behavior, and meningoencephalitis. Some individuals with this disease remain asymptomatic for long periods. ${ }^{1-3}$ Human anti-cysticercus antibodies have been described as an indicator of cysticercosis as well as of the prevalence of Taenia solium tapeworm infections. ${ }^{4,5}$

Immune diagnosis and clinical manifestations of human cysticercosis have been recognized in some countries in southern Africa. ${ }^{6-9}$ In Mozambique, no data on human cysticercosis in hospitalized patients have been reported, but as of 1990 only one case detected during an autopsy was reported in the literature. ${ }^{10}$

Taenia solium infection in pigs is known to be endemic in some regions of Mozambique. ${ }^{11}$ In Maputo, free-ranging pigs are not normally raised and pig cysticercosis is not recognized. The aim of this study was to determine the seroprevalence of human cysticercosis in a cohort of the urban population where there is poor environmental sanitation in places overcrowded by refugees from other regions of Mozambique.

\section{MATERIALS AND METHODS}

Study design. A serologic survey for cysticercosis was undertaken from January to June 1993 among patients or visitors entering the Central Hospital of Maputo premises. Four hundred eighty-nine individuals with no clinical diagnosis of cysticercosis were selected from three groups of people at the Central Hospital of Maputo. Two hundred twenty-two were blood donors and patients from the Department of Orthopedics (BD/DO), 148 were patients from the Department of Neurology (DN), and 119 were patients from the Department of Psychiatry (DP). We combined the BD and DO groups because they presented at the hospital with no mental, psychiatric, or infectious conditions. All individuals five years of age or older that presented to these departments were invited to participate in the study.

The protocol for this study was reviewed and approved by the Scientific Board of the Public Health Department of the Universidade Nova de Lisbon, the Scientific Board of the Universidade Eduardo Mondlane, and the Ministry of Health of Mozambique.
Data collection. Data were collected using a standardized questionnaire that included age, sex, place of birth, occupation, educational level, place of residence (urban, suburban, or rural), household characteristics (adobe, hut, shack, apartment), source of water supply (potable water, piped water, or superficial water), diet habits, toilet facilities (toilet, latrine), income, religious practices, and clinical manifestations compatible with neurocysticercosis. We grouped the factors source of water supply, toilet facilities, and household characteristics into a single variable called sanitary facilities with three levels. In the first level (high sanitary conditions), participants lived in an apartment and had potable water and a toilet. In the second level (median sanitary conditions), they did not have a toilet but did have a latrine. In the third level (low sanitary conditions), they lived in a shack and lacked a toilet and/or potable water.

Serum samples. Serum was separated by centrifugation and stored at $-20^{\circ} \mathrm{C}$ in a cryotube (Nunc, Roskilde, Denmark) until used.

Enzyme-linked immunosorbent assay. The serology for cysticercosis antibodies was performed using a commercial ELISA (Melotest for cysticercosis: Melotec SA, Barcelona, Spain). It had a sensitivity of $96 \%$ and a specificity of $97 \%$ based on a study of 156 sera, of which 23 were from patients confirmed to have cysticercosis and 136 were from healthy people used as control group. The manufacturer indicated the possibility of cross-reactivity with serum from patients with hydatidosis. Human hydatidosis is not recognized as a public health problem in Mozambique. Sera with an optical density ratio (sample:control) $\geq 1.2$ were considered positive, those between 0.9 and 1.2 were considered indeterminate, and those $\leq 0.9$ were considered negative.

Data analysis. Data from questionnaires and laboratory results were stored and analyzed using the computer package Epi-Info (version 6) (USD Corp., Stone Mountain, GA) and the chi-square test, Fisher's exact test, and the F and $\mathrm{K}$ tests.

\section{RESULTS}

Demographic, environmental, and behavioral characteristics. Of 489 individuals that participated in the survey, $150(30.7 \%)$ were females and $339(69.3 \%)$ were males. The 
TABLE 1

Population characteristics among the three groups*

\begin{tabular}{|c|c|c|c|c|c|c|c|}
\hline \multirow[b]{2}{*}{ Group $\dagger$} & \multicolumn{2}{|c|}{ Males } & \multicolumn{2}{|c|}{ Females } & \multirow{2}{*}{$\begin{array}{l}\text { Average age } \\
\text { (years) }\end{array}$} & \multirow[b]{2}{*}{ Sanitary facilities } & \multirow[b]{2}{*}{ Religion§ } \\
\hline & No. & $\%$ & No. & $\%$ & & & \\
\hline $\mathrm{BD} / \mathrm{DO}$ & 187 & 84.2 & 35 & 15.8 & 28.7 & $1=50,2=58,3=114$ & $a=135, b=81, c=6$ \\
\hline $\mathrm{DN}$ & 86 & 58.1 & 62 & 41.9 & 34.4 & $1=73,2=39,3=35$ & $a=42, b=89, c=16$ \\
\hline DP & 66 & 55.5 & 53 & 44.5 & 33.6 & $1=69,2=28,3=21$ & $a=50, b=56, c=12$ \\
\hline$P$ & \multicolumn{4}{|c|}{$<0.0001$} & $<0.001$ & $<0.0001$ & $<0.005$ \\
\hline
\end{tabular}

male to female ratio was 5.3:1 in the BD/DO group, $1.3: 1$ in the DN group, and $1.2: 1$ in the DP group $(P<0.001)$. There were 20 children (4\%) less than 10 years of age. The age distribution ranged from five to 75 years, with a mean age of 31.7 years (95\% confidence limit $=30.5-32.8$ years). People in BD/DO group were the youngest.

Almost $88 \%$ (429) of the individuals were born in the southern provinces of Mozambique. The BD/DO group lived in Maputo for an average of 17 years and the DN and DP groups lived there for an average of 23 years $(P<0.001)$.

Individuals without a profession and those unemployed with low qualifications and without any income were the most common group $(31.3 \%)$, followed by skilled workmen $(30.5 \%)$. Skilled workmen were more representative in the $\mathrm{BD} / \mathrm{DO}$ group (36\%) and housewives and the unemployed were most common in the DN (36.5\%) and DP (34.5\%) groups.

Only $2 \%$ had a high school education and $9.5 \%$ lacked elementary instruction. Most $(65.2 \%)$ had completed primary school and the first year of secondary school. The DP group had the highest education level $(P<0.05)$ and more than half lived in good sanitary conditions.

The BD/DO group lived in the poorest environmental and sanitary conditions $(P<0.001)$ (Table 1$)$. One hundred ninety-two people $(39.4 \%)$ lived in apartments or cottages with toilet facilities and potable water supplied. One hundred seventy people $(34.8 \%)$ did not have any toilet facilities. Three hundred eighty-seven individuals $(79.6 \%)$ occasionally consumed pork (including four Moslems) and 92 persons had never eaten pork $(20.4 \%)$.

We did not find any predominant religion in the study population. Two hundred twenty-six $(46.4 \%)$ individuals reported to be Catholics or Evangelists, 138 (28.5\%) were Messianic or Syncretic, 88 (18.1\%) were Animists, and 34 (7\%) were Hindus or Moslems (Table 2).

Serologic results. Of 489 serum samples tested, 59
(12.1\%) were positive for anti-cysticercosis antibodies. Seropositivity rates were similar in the $\mathrm{BD} / \mathrm{DO}(14.9 \%)$ and DN (11.5\%) groups, and lower in the DP group (7.6\%) (Table 2). Seroprevalence was highest among those 20-29 and 40-49 years of age.

Sixteen of 17 people in the DN group with cysticercosis antibodies had clinical manifestations (headaches, seizures, epilepsy, paraplegia, and dementia) compatible with neurocysticercosis, as did all nine antibody-positive individuals in the DP group. Five of 33 individuals in the BD/DO group with cysticercosis antibodies reported only headaches. There were no significant differences in the clinical signs between the seropositive and seronegative groups.

Comparison between cysticercosis-seropositive and -seronegative individuals. We detected no differences between the seropositive and seronegative groups in terms of qualifications, occupation, religion, travel history, and diet habits (including pork consumption), and place of birth.

Among those with anti-cysticercosis antibodies, the most important factors associated with seropositivity were a poor water supply $(P<0.0001)$ poor or no toilet facilities $(P<$ $0.006)$, and poor housing $(P<0.001)$ compared with those negative for anti-cysticercosis antibodies. The seronegative group had more skilled workmen $(P<0.05)$, the average age was higher $(P<0.001)$, and they had lived for a longer time in Maputo $(P<0.05)$ (Table 3$)$.

\section{DISCUSSION}

Typical houses in Mozambique are made of wood and cane and consist of one or two rooms. The social life of the family normally occurs outside the home. Public sanitary facilities are limited and largely deficient. Medical care is available in health centers, but a large number of people make use of traditional medical care providers (faith healers).

TABLE 2

Results of the serologic tests*

\begin{tabular}{|c|c|c|c|c|c|c|c|c|}
\hline & \multicolumn{8}{|c|}{ Group } \\
\hline & \multicolumn{2}{|c|}{$\mathrm{BD} / \mathrm{DO}$} & \multicolumn{2}{|c|}{ DN } & \multicolumn{2}{|c|}{ DP } & \multicolumn{2}{|c|}{ Total } \\
\hline & No. & $\%$ & No. & $\%$ & No. & $\%$ & No. & $\%$ \\
\hline Positive & 33 & 14.9 & 17 & 11.5 & 9 & 7.6 & 59 & 12.1 \\
\hline Indeterminate & 19 & 8.5 & 11 & 7.4 & 3 & 2.5 & 33 & 6.7 \\
\hline \multirow[t]{2}{*}{ Negative } & 170 & 76.6 & 120 & 81.1 & 107 & 89.9 & 397 & 81.2 \\
\hline & 222 & 100 & 148 & 100 & 119 & 100 & 489 & 100 \\
\hline
\end{tabular}

* BD/DO = blood donors and Department of Orthopaedics patients; DN = Department of Neurology patients; DP = Department of Psychiatry patients. 
TABLE 3

Comparison between cysticercosis-seropositive and -seronegative individuals*

\begin{tabular}{|c|c|c|c|c|c|c|c|c|c|c|c|}
\hline & \multicolumn{2}{|c|}{ More skilled } & \multicolumn{2}{|c|}{ Less skilled } & \multicolumn{2}{|c|}{ HSC } & \multicolumn{2}{|c|}{ MSC } & \multicolumn{2}{|c|}{ LSC } & \multirow{2}{*}{$\begin{array}{l}\text { Average years living } \\
\text { in Maputo } \dagger\end{array}$} \\
\hline & No. & $\%$ & No. & $\%$ & No. & $\%$ & No. & $\%$ & No. & $\%$ & \\
\hline $\begin{array}{l}\text { Seropositive } \ddagger \\
\quad(\mathrm{n}=59)\end{array}$ & 6 & 10.2 & 53 & 89.8 & 15 & 25.9 & 10 & 17.2 & 33 & 56.9 & $\begin{array}{c}16 \\
(12.6-19.8)\end{array}$ \\
\hline $\begin{array}{l}\text { Seronegative } \\
\quad(\mathrm{n}=396)\end{array}$ & 82 & 20.7 & 314 & 79.3 & 16 & 42.7 & 11 & 28.0 & 116 & 29.3 & $\begin{array}{c}21 \\
(19.9-22.5)\end{array}$ \\
\hline$\chi^{2}$ & \multicolumn{2}{|c|}{3.66} & & & & & \multicolumn{2}{|c|}{17.48} & & & $F=7.39$ \\
\hline$P$ & \multicolumn{2}{|c|}{0.05} & & & & & \multicolumn{2}{|c|}{$<0.0001$} & & & $P=0.006$ \\
\hline
\end{tabular}

$* \mathrm{HSC}=$ high sanitary conditions; $\mathrm{MSC}=$ medium sanitary conditions; $\mathrm{LSC}=$ low sanitary condition

$\dagger$ Values in parentheses are $95 \%$ confidence intervals.

$\ddagger$ It was not possible to classify the sanitary condition of one person from the seropositive group

In Mozambique, cysticercosis is usually not considered in the differential diagnosis of a neurologic illness. Fifty-nine of the 489 people tested were positive for anti-cysticercosis antibodies. Twenty-six were young people who had lived for a short time in Maputo $(P<0.001)$. People living in poorest sanitary conditions had the highest seropositivity rate $(P<$ $0.001)$. More than half $(52.5 \%)$ of those with serologic evidence of cysticercosis had some sign suggestive of neurocysticercosis such as headaches, seizures, epilepsy, paraplegia, and dementia.

Since Mozambican traditional culture is deeply rooted in magical religious beliefs, people frequently make use of a traditional spiritual care provider before seeking any medical advice. This factor may influence the differences in the seropositivity rates among the three groups. Differences in education level among the three groups can be explained by the difference in the sex ratio. Traditionally, women in Mozambique receive less formal education.

This preliminary study shows that human cysticercosis should be considered in the differential diagnosis of neurologic disturbances, even among a population where pig cysticercosis is not observed. Appropriate advice on personal hygiene and proper environmental control measures are crucial if high standards of public health are to be achieved. This study was not representative of the entire population of Maputo because it was a hospital-based study. Although the results of the current study reflect sampling bias, it represents a first step in promoting future actions. We observed an overall seroprevalence of $12 \%$, which is higher compared with others studies in Africa ${ }^{12-14}$ (Table 4).

It is important to carry out additional epidemiologic studies and develop cheap and reliable techniques for the diagnosis of human cysticercosis. Moreover, it is essential to improve the meat quality standards and give appropriate training to the meat inspection health officers.

TABLE 4

Prevalence of cysticercosis in other studies in Africa

\begin{tabular}{lcc}
\hline \multicolumn{1}{c}{ Country } & $\begin{array}{c}\text { Seroprevalence by } \\
\text { ELISA }\end{array}$ & Reference \\
\hline South Africa & $5.5 \%$ & 6 \\
Zimbabwe & $12 \%$ & 12 \\
Reunion & $8.2 \%$ & 13 \\
Benin & $3.5 \%$ & 14 \\
\hline
\end{tabular}

Acknowledgments: We thank all the individuals who participated in the study, and the staff of the Faculty of Medicine of Eduardo Mondlane and the Hospital Central de Maputo, Mozambique for allowing us to use their facilities and patient serum samples. We also thank Dr. Helena Ângelo (Instituto Nacional de Saude Dr. Ricardo Jorge, Lisbon, Portugal) for kindly allowing us to use her diagnostic laboratory, and Drs. Alcione Trinca, Cristina Furtado, Eduardo Medeiros, João Falcato, Madalena Cabeçadas, and Vasco Mendes, and Professors J. L. Castanheira and J. A. Cruz e Silva for expert advice.

Financial support: This work was supported by Fundação para a Ciência e Technologia PRAXIX XXI, Master Scholarship no. 3370/92 - ID.

Authors' addresses: Manuela Vilhena, Urbanização da Portela, Rua Palmira Bastos No. 13, $11^{\circ}$ Esq, 2685 Portela LRS, Portugal. Manuela Santos, Rua de Nashingweia, 168, Maputo, Mozambique. Jorge Torgal, Faculdade de Ciências Médicas, Departamento de Saude Publica, Universidade Nova de Lisboa, Campo de Santana 130, 1198 Lisbon, Portugal.

\section{REFERENCES}

1. Schantz PM, Cruz M, Sarti E, Pawlowski ZS, 1993. Potential eradicability of taeniasis and cysticercosis. Bull Pan Am Health Organ 27: 397-403.

2. Crimmins D, Collignon PJ, Dwyer D, Danta G, 1990. Neurocysticercosis: an under-recognised cause of neurological problems. Med J Aust 152: 434-438.

3. Dansey RD, Hay M, Cowie RL, 1992. Seizures and neurocysticercosis in black men. $S$ Afr Med $J$ 81: 424-425.

4. Camacho SD, Ruiz AC, Beltran MU, Willms K, 1990. Serology as an indicator of Taenia solium tapeworm infections in a rural community in Mexico. Trans $R$ Soc Trop Med Hyg 84: 563-566.

5. Schantz PM, Sarti-Gutierrez E, 1989. Diagnostic methods and epidemiologic surveillance of Taenia solium infection. Acta Leiden 57: 153-163.

6. Mason P, Houston S, Gwanzura L, 1992. Neurocysticercosis: experience with diagnosis by ELISA serology and computerized tomography in Zimbabwe. Cent Afr J Med 38: 149154.

7. Sacks LV, Berkowitz I, 1990. Cysticercosis in an urban South African community: prevalence and risk factors. Trop Gastroenterol 11: 30-33.

8. Sacks LV, Zini D, Klein C, Saffer D, Wadee AA, 1989. The use of an ELISA for the diagnosis of cysticercosis in epileptic patients. S Afr J Epidemiol Infect 4: 50-52.

9. Thomson AJ, 1993. Neurocysticercosis-experience at teaching hospitals of the University of Cape Town. S Afr Med J 83: 332-334.

10. Serra L, 1968. Um caso coincidente de raiva e cisticercose no homem. Vet Moçambicana. Lourengo Marques 1: 21-26.

11. Vilhena M, Bouza M, 1994. Estudo piloto sobre teniase e cis 
ticercose humana no distrito de Tete. Rev Med Moçambique 5: 6-9.

12. Shasha W, Pammenter MD, 1991. Sero-epidemiological studies of cysticercosis in school children from two rural areas of Transkei, South Africa. Ann Trop Med Parasitol 85: 349-355.

13. Michault A, Duval G, Bertil G, Foho G, 1990. Seroepidemio- logical study of cysticercosis on Reunion Island. Bull Soc Pathol Exot 83: 82-92.

14. Adjide CC, Bouteille B, Josse R, Adjide-Szmictt V, Avode DG, Dumas M, 1996. Seroprevalence of cysticercosis in the lakeside community of Vekky, Atlantic District (Benin). Bull Soc Pathol Exot 89: 24-29. 